\title{
Dual-band Dual-polarized Antenna for mm-Wave 5G Base Station Antenna Array
}

\author{
Zeeshan Siddiqui ${ }^{1}$, Marko Sonkki ${ }^{1}$, Jiangcheng Chen $^{1}$, Markus Berg ${ }^{1}$, Marko E. Leinonen ${ }^{1}$, Aarno Pärssinen ${ }^{1}$ \\ ${ }^{1}$ Centre for Wireless Communications, University of Oulu, Oulu, Finland, email: zeeshan.siddiqui@oulu.fi
}

\begin{abstract}
A dual-band dual-polarized antenna suitable for 5G millimeter-wave base station antenna array is presented in this paper. It operates on all the commercial millimeter wave frequencies allotted in 5G NR from $24.25 \mathrm{GHz}$ up to $40 \mathrm{GHz}$. The antenna is based on a novel stacked square ring patches arrangement to achieve wide dual band and stable radiation pattern. The antenna offers a sharp roll-off and a filter like response between the operating bands due to the strongly coupled resonators. Antenna design principle and simulated performance are discussed in detail. The $-10 \mathrm{~dB}$ impedance bandwidth of the lower band starts from $24.25 \mathrm{GHz}$ to $29.5 \mathrm{GHz}$ while the higher band covers the $37 \mathrm{GHz}$ to $40 \mathrm{GHz}$. The realized gain remains stable between 5 to $6 \mathrm{dBi}$ at all the operating frequencies. The isolation between the ports and cross-polar discrimination remain better than $20 \mathrm{~dB}$ in all the covered frequency range.
\end{abstract}

Index Terms-5G NR, base station antenna array, dualband dual-polarized antenna, filtenna.

\section{INTRODUCTION}

The trial and deployment of 5G NR (New Radio) networks have already started in different regions of the globe. Millimeter-wave (mm-Wave) communication is one of the key enabling technologies offered by the $5 \mathrm{G}$ for high data rate and low-latency applications [1]. There are several challenges on the antenna front from electrical, mechanical and commercial aspects. Antennas are required to cover wide bandwidths with reasonable and stable pattern preferably supporting dual polarizations. They need to be low profile and meet the antenna-in-package (AIP) considerations [2]. After all, the solution should be low cost to be commercially competitive. For mm-Wave communications, antenna arrays are required at the base stations in order to meet the link budget requirements. The array issues, like array geometry and its integration, mutual coupling and grating lobe also need to be considered during the antenna array unit cell design.

Few different antenna array solutions have been demonstrated to meet the mm-Wave base station requirements. For example, phased array horn antenna is presented to cover the $28 \mathrm{GHz}$ band [3] and a Vivaldi antenna array is demonstrated to cover the $26 \mathrm{GHz}$ band [4]. However, microstrip patch antennas are preferred due to their low profile, ease of integration and low cost. There are associated challenges like narrow bandwidth, surface waves and losses due to the inherent nature of microstrip antennas. Patch stacking is a well-known technique to enhance the bandwidth of microstrip antennas. A stacked patch antenna for mm-Wave base station is presented to cover the $28 \mathrm{GHz}$ band [5]. For

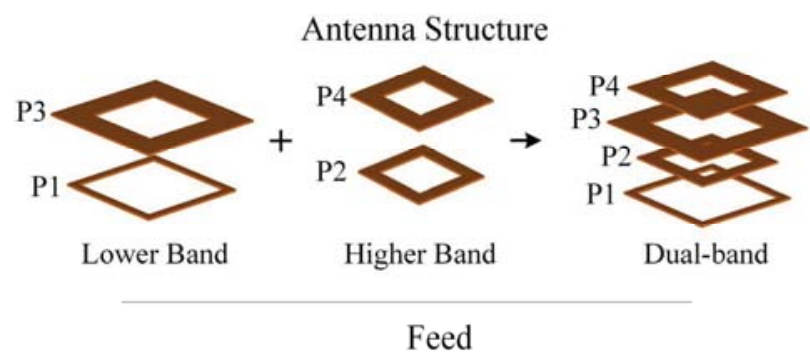

Fig. 1. Antenna structure for wide, dual band operation.

mobile terminals, a dual-band dual-polarized stacked patch antenna with parasitic elements is demonstrated at $28 \mathrm{GHz}$ and $39 \mathrm{GHz}$ bands [6].

In this paper, a dual-band dual-polarized antenna covering three, $26 \mathrm{GHz}, 28 \mathrm{GHz}$ and $39 \mathrm{GHz} 5 \mathrm{G} \mathrm{NR}$ mm-Wave bands is presented. The lower band, from $24.25 \mathrm{GHz}$ to $29.5 \mathrm{GHz}$, covers the $26 \mathrm{GHz}$ and $28 \mathrm{GHz}$ bands with $19.5 \%$ of fractional bandwidth. Over $8 \%$ of fractional bandwidth is achieved at $39 \mathrm{GHz}$, higher band from $37 \mathrm{GHz}$ to $40 \mathrm{GHz}$. In the following, antenna design and its operating mechanism is discussed in Section II. The simulated results are discussed in Section III followed by the conclusion and future work in Section IV.

\section{ANTENNA DESIGN}

Generally, the stacking technique is utilized to obtain either wide-band [7] or dual-band [8] operation. However, the proposed antenna design utilizes the stacking approach to achieve a wide and dual-band operation as discussed below.

\section{A. Antenna Element Design}

The proposed antenna is based on four stacked squarering patches arranged in a novel configuration to achieve wide dual-band with a reasonable and stable radiation pattern as shown in Fig.1. A square-ring patch is similar to a square patch with a portion around its center being removed. Geometrically, it is an intermediate configuration between a printed loop and solid patch. The ring geometry offers additional parameter, inner length, to control resonant frequency, impedance and bandwidth of the patch. The ratio of the inner to the outer length of the patch influence its resonant frequency. The outer length remains between quarter to half-effective wavelength for the resonance [9]. 


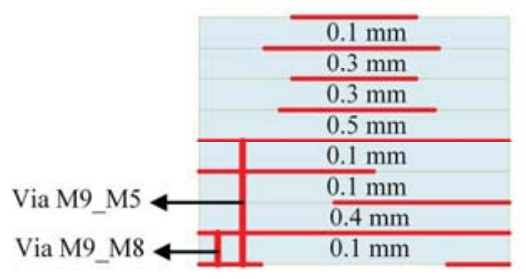

M1: Patch 4

M2: Patch 3

M3: Patch 2

M4: Patch 1

M5: Antenna Ground M6: Stripline Feed_1 M7: Stripline Feed 2 M8: Feed_Ground M9: Microstrip Feed

Fig. 2. Stack of the proposed dual band dual polarized antenna with aperture coupled feed.

The selection of appropriate substrate material for the patch antenna needs consideration of multiple issues. Generally, a low dielectric constant and low loss material is preferred to have better bandwidth and efficiency. On the other hand, for AIP, the chosen substrate should comply with the multilayer fabrication requirements. Here, Panasonic Megtron 7 is selected for the antenna design for its ultra-low loss and multi-layer properties [10]. It has dielectric constant around 3.35 and dissipation factor of 0.004 at the operating frequencies.

For an antenna array, element size plays a vital role in its far-field performance. Generally, the spacing between the elements is less than half wavelength at the highest frequency to avoid grating lobes. In the presented case, the operating bands are wide apart and a compromise is required in the selection of unit cell size. It is kept at $5 \mathrm{~mm}, \lambda / 2$ at $30 \mathrm{GHz}$, to have sufficient gain at the lower band, although the scan range is reduced at the higher frequency band.

The antenna is designed targeting the mm-Wave $5 \mathrm{G} \mathrm{NR}$ bands over the selected substrate material and the cell size as discussed earlier. The design is initiated by observing the performance of a square patch and influence of inner cutout through parametric study at the lower frequency band. The stacking technique is utilized by introducing a second resonator to enhance the bandwidth. Apart from the ring dimensions, the other critical parameter in stacked configuration is the spacing among elements and feed, as the coupling strongly depends on it. Generally, for a stacked patch antenna configuration, the lower patch is strongly coupled to the feed, over-coupled. The introduction of second patch effectively impedance match the antenna [11].

In the presented work, a second set of coupled resonators operating at the higher frequency band is introduced to achieve wide, dual-band operation. The initial design of the higher band stacked patch configuration follows the same procedure as discussed earlier. The two stacked coupled resonators operating at different frequency bands are merged in an alternate resonance configuration as depicted in Fig.1. The detuning of the resonators due to the loading by the other set of resonators can be corrected by optimizing the patch dimensions. It is observed that an alternate placement of patch antenna yields the best results in the discussed configuration. It can be lower band antenna resonator first, followed by the higher band resonator or otherwise depending on the antenna feed and coupling requirements.

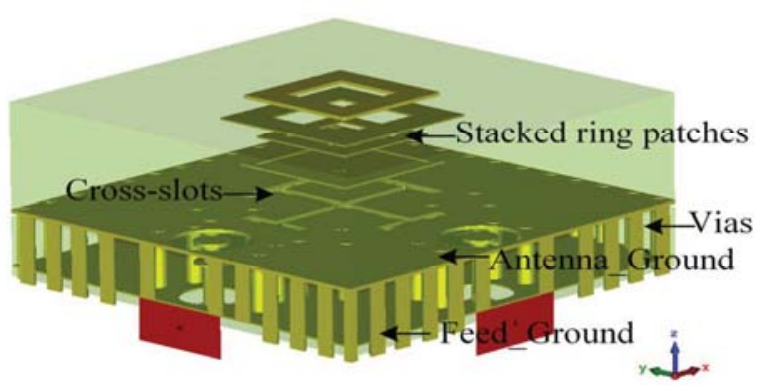

(a)

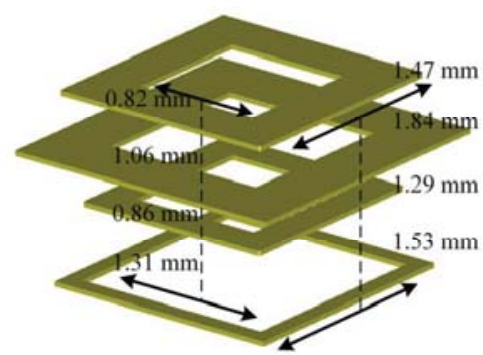

(b)

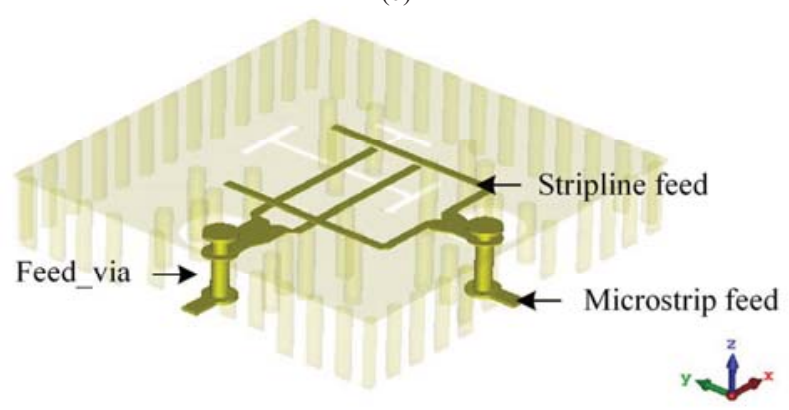

(c)

Fig. 3. CST MWS simulation model (a) Perspective view (b) zoomed stacked patches (c) feed network

The resonators are strongly coupled to the respective adjacent resonator over the frequency spectrum. This gives a sharp roll-off and passband filter like response, particularly at frequencies in between the two operating bands. In this view, the antenna may be considered as a filtenna, easing the load on transceiver filters. In the following, integration of the antenna with the feed and related issues are discussed.

\section{B. Aperture Coupled Antenna Design}

The stack of the proposed antenna with nine metal layers is shown in Fig.2. The top five layers are for the radiating structure and its ground while the remaining are for the feed network. To simplify the fabrication process, all the vias are connecting M5 to M9 with $0.2 \mathrm{~mm}$ diameter. An additional $0.1 \mathrm{~mm}$ via connecting M8 to M9, placed for the ground connection. The simulation model is shown in Fig.3 with all the simulations being carried out in CST Microwave Studio. The model, with connector excluded, is excited by the waveguide ports. 


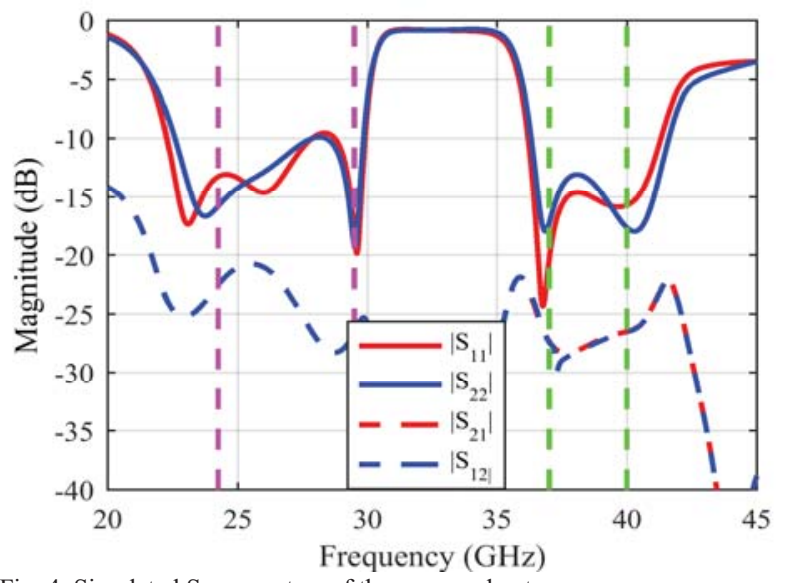

Fig. 4. Simulated S-parameters of the proposed antenna.

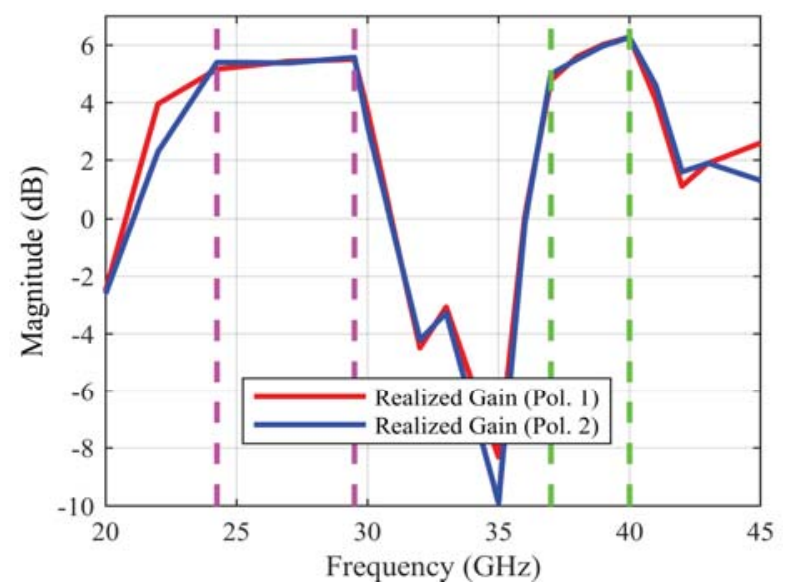

Fig. 5. Realized gain vs. frequency plot of the proposed antenna

Although the operation of the discussed antenna is independent of feed structure, the matching and far-field performance is influenced by the feeding technique. Noncontact feeding techniques, like proximity coupling and aperture coupling are preferred at mm-wave frequencies, as they do not deteriorate the radiation pattern [11].

In the presented work, aperture coupling is used to feed the antenna as shown in the simulation model. The feed network is isolated from the radiating elements and can be independently optimized. Considering the AIP, stripline feed network is adopted, although it needs more substrate volume than microstrip structure. Also, parallel plate modes [12] appeared at the operating frequencies, due to stripline structure. Shorting vias are placed around the slot aperture to effectively suppress them. Their positions are optimized through iterative simulations. To shield the signal, a coaxial like structure is emulated by placing grounded vias around the feed via [13]. At the bottom layer, a microstrip line is placed to feed the antenna. Microstrip to stripline transition is optimized by tuning the circular ground cutouts and the spacing between the signal and the ground vias. In the following section, simulation results on the antenna's performance and behavior are discussed.

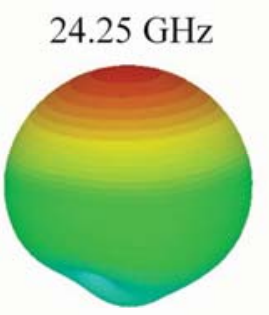

$5.3 \mathrm{~dB}$

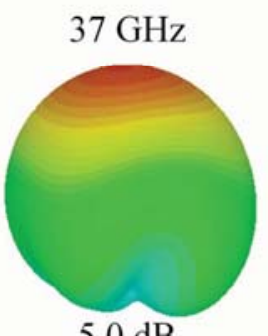

$5.0 \mathrm{~dB}$
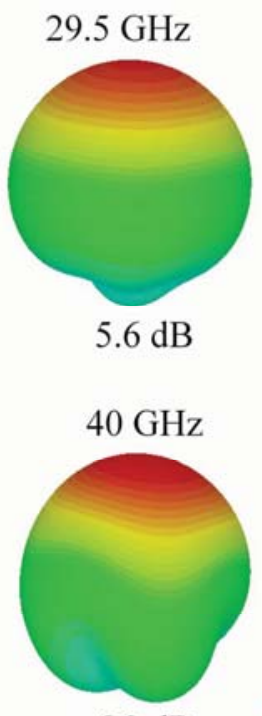

$6.3 \mathrm{~dB}$
dB

6

5.25

4.5

3.75

3

2.25

1.5

0.75

0

$-4.25$

$-8.5$

$-12.8$

$-17$

$-21.2$

$-25.5$

$-29.8$

$-34$

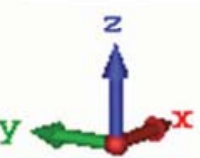

Fig. 6. 3D radiation pattern of the proposed antenna at different frequencies.

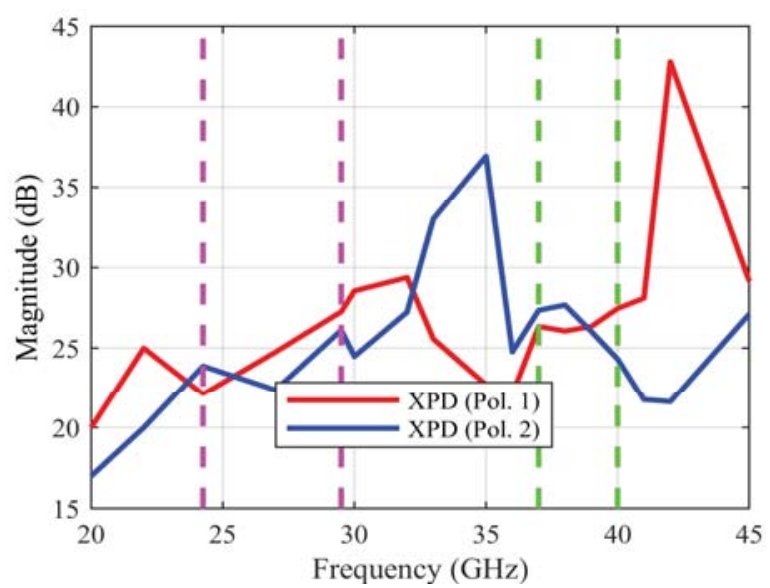

Fig. 7. Cross-polar discrimination, XPD plot of the proposed antenna.

\section{RESUlTS AND DisCUSSION}

Fig. 4. presents the simulated S-parameters of the proposed antenna element. $-10 \mathrm{~dB}$ impedance bandwidth is achieved at both the lower and higher bands. The important filtering and sharp roll-off feature can be observed in the plot as discussed earlier. A fine-tuning of the spacing between the ring patches and their dimensions can further improve the matching around $28 \mathrm{GHz}$. The feeding network and the slot aperture are non-identical causing minor deviation between the ports matching. The isolation between the ports remains below the $-20 \mathrm{~dB}$ and $-25 \mathrm{~dB}$ in both the lower and higher bands respectively.

The realized gain against the frequency is plotted in Fig. 5. The gain is stable between 5 to $6 \mathrm{dBi}$ over both the studied bands. Further improvement around $37 \mathrm{GHz}$ can be achieved by fine adjustment of the spacing between the elements. A significant drop in gain can also be observed between the 
operating bands. Fig. 6 shows the 3D radiation pattern at different operating frequencies. A stable and almost symmetric radiation pattern is achieved. The feeding slot aperture's resonance is close to the higher band, which might be affecting the radiation pattern. Another important far-field parameter is the cross-polar discrimination (XPD) being plotted in Fig. 7. It is better than $20 \mathrm{~dB}$ and $25 \mathrm{~dB}$ in both the lower and higher bands respectively. The minor differences between the two polarizations are likely due to the different electrical length of feeding slot aperture.

\section{CONCLUSION}

This paper presented a multilayer, dual-band dual polarized antenna for $\mathrm{mm}-$ Wave base station arrays, covering 5G NR bands from $24.25 \mathrm{GHz}$ to $40 \mathrm{GHz}$. A novel antenna configuration is introduced by stacking antenna elements in an alternating resonance order. Electrically, the demonstrated antenna shows wide dual bands, reasonable gain, isolation and XPD with stable radiation pattern at all the operating frequencies. From the fabrication point of view, it is a simple geometry meeting the requirements of common commercial fabrication capabilities with minimum via requirements. On the other hand, it has a maximum of two additional metal layers in comparison to the existing solutions. Next, the designed antenna will be fabricated and measured.

\section{ACKNOWLEDGMENT}

This work has been partly funded by Business Finland project 5G Test Network Plus (5GTN+), and partly by the Academy of Finland project 6Genesis Flagship (grant no. 318927).

\section{REFERENCES}

[1] Binqi Yang, Zhiqiang Yu, Ji Lan, Ruoqiao Zhang, Jianyi Zhou and Wei Hong, "Digital Beamforming-Based Massive MIMO Transceiver for 5G Millimeter-Wave Communications," IEEE Transactions on Microwave Theory and Techniques, vol. 66, no. 7, pp. 3403-3418, 2018.

[2] Duixian Liu, Xiaoxiong Gu, Christian W Baks and Alberto ValdesGarcia, "Antenna-in-Package Design Considerations for Ka-Band 5G Communication Applications," IEEE Transactions on Antennas and Propagation, vol. 65, no. 12, pp. 6372-6379, 2017.

[3] Mark TAN Y. C, Guan Hong NG and Roger TAY Y. S, "A 2-By-2 Sub-Array for Scalable 28GHz mmWave Phased Array Horn Antenna in 5G Network," in Proc. 2019 13th European Conference on Antennas and Propagation (EuCAP), 2019.

[4] Shuangshuang Zhu, Haiwen Liu, Zhijiao Chen and Pin Wen, "A Compact Gain-Enhanced Vivaldi Antenna Array With Suppressed Mutual Coupling for $5 \mathrm{G}$ mmWave Application," IEEE Antennas and Wireless Propagation Letters, vol. 17, no. 5, pp. 776-779, 2018.

[5] In-June Hwang, Hye-Won Jo, ByungKuon Ahn, Ju-ik Oh and JongWon Yu, "Cavity-backed Stacked Patch Array Antenna with Dual Polarization for mmWave 5G Base Stations," in Proc. 2019 13th European Conference on Antennas and Propagation (EuCAP), 2019.

[6] Manoj Stanley, Yi Huang, Hanyang Wang, Hai Zhou, Ahmed Alieldin, Sumin Joseph, Chaoyun Song and Tianyuan Jia."A DualBand Dual-Polarised Stacked Patch Antenna for $28 \mathrm{GHz}$ and $39 \mathrm{GHz}$ 5G Millimetre-Wave Communication," in Proc. 13th European Conference on Antennas and Propagation (EuCAP), 2019.

[7] S.D Targonski, R.B Waterhouse and D.M Pozar, "Design of wideband aperture-stacked patch microstrip antennas," IEEE Transactions on Antennas and Propagation, vol. 46, no 9, pp. 1245-1251, 1998.
[8] "A dual-band circularly polarized aperture-coupled stacked microstrip antenna for global positioning satellite," IEEE Transactions on Antennas and Propagation, vol. 45, nro 11, pp. 1618-1625, 1997.

[9] P.M Bafrooei, L Shafai, "Characteristics of single- and double-layer microstrip square-ring antennas," IEEE Transactions on Antennas and Propagation, vol. 47, no. 10, pp. 1633-1639, 1999.

[10] Panasonic multi-layer circuit board materials, Available: https:// industrial.panasonic.com/ww/products/electronic-materials/circuitboard-materials/megtron/megtron7.

[11] R. B Waterhouse, Microstrip Patch Antennas: A Designer's Guide, Boston, Springer US, 2003.

[12] Renato Rimolo-Donadio, J Supper, T Winkel, H Harrer and C Schuster, "Analysis and Mitigation of Parasitic Mode Conversion for Microstrip to Stripline Transitions," IEEE Transactions on Electromagnetic Compatibility, vol. 54, no. 2, pp. 495-498, 2012.

[13] A Stark, A.F Jacob, "A Broadband Vertical Transition for MillimeterWave Applications," in Proc. 38th European Microwave Conference, 2008 . 